\title{
Risk-based constraints with correlated uncertainties for the optimal operation of an energy community
}

This paper was downloaded from TechRxiv (https://www.techrxiv.org).

\section{LICENSE}

CC BY 4.0

SUBMISSION DATE / POSTED DATE

$16-06-2021 / 18-11-2021$

\section{CITATION}

Dolányi, Mihály; Bruninx, Kenneth; Toubeau, Jean-François; Delarue, Erik (2021): Risk-based constraints with correlated uncertainties for the optimal operation of an energy community. TechRxiv. Preprint. https://doi.org/10.36227/techrxiv.14790252.v2

$\mathrm{DOI}$

10.36227/techrxiv.14790252.v2 


\title{
Risk-based constraints with correlated uncertainties for the optimal operation of an energy community
}

\author{
Mihaly Dolanyi, Kenneth Bruninx, Jean-François Toubeau, and Erik Delarue
}

\begin{abstract}
This paper formulates an energy community's centralized optimal bidding and scheduling problem as a time-series scenario-driven stochastic optimization model, building on reallife measurement data. In the presented model, a surrogate battery storage system with uncertain state-of-charge (SoC) bounds approximates the portfolio's aggregated flexibility. First, it is emphasized in a stylized analysis that risk-based energy constraints are highly beneficial (compared to chance-constraints) in coordinating distributed assets with unknown costs of constraint violation, as they limit both violation magnitude and probability. The presented research extends state-of-the-art models by implementing a worst-case conditional value at risk (WCVaR) based constraint for the storage SoC bounds. Then, an extensive numerical comparison is conducted to analyze the trade-off between out-of-sample violations and expected objective values, revealing that the proposed WCVaR based constraint shields significantly better against extreme out-of-sample outcomes than the conditional value at risk based equivalent. To bypass the non-trivial task of capturing the underlying time and assetdependent uncertain processes, real-life measurement data is directly leveraged for both imbalance market uncertainty and load forecast errors. For this purpose, a shape-based clustering method is implemented to capture the input scenarios' temporal characteristics.
\end{abstract}

Index Terms-Energy community, Optimal bidding, Stochastic optimization, Risk-based constraints, Temporal correlation

\section{NOMENCLATURE}

Parameters

$\Delta \widetilde{d}_{t, \theta}^{R T}$ Real-time forecast error at time $t$, in scenario $\theta$

$\eta^{c h}{ }_{,} \eta^{\text {dch }}$ Charging and discharging efficiency (-)

${\underset{\sim}{\lambda_{t}}}_{\lambda_{t, \theta}} \quad$ Day-ahead energy market price at time $t$

$\tilde{\lambda}_{t, \theta}^{R T} \quad$ Real-time imbalance price at time $t$, scenario $\theta$

$\bar{E} \quad$ Energy capacity of the ESS (MWh)

$\bar{P} \quad$ Power capacity of the ESS (MW)

$\underline{\mathrm{a}}^{D A}, \bar{a}^{D A}$ Upper and lower limit of the community's day-ahead market position

$\underline{\mathrm{a}}^{R T}, \overline{\mathrm{a}}^{R T}$ Upper and lower limit of the community's real-time market position

$\mathrm{d}_{t}^{D A} \quad$ Day-ahead demand of the community at time $t$

$\mathrm{OPEX}^{D A}$ Operational expenditure of the distributed storage

Sets and indices

$\pi \in \Pi$ Load forecast error scenarios

$\theta \in \Theta$ Imbalance price scenarios

$d \in D$ Number of simulated days in the out-of-sample tests

$k \in K$ Clusters of load deviation scenarios

$n \in N$ Number of scenarios within cluster $\mathrm{k}$

$t \in T$ Time steps

Variables

$\Delta \widetilde{a}_{t, \theta}^{R T}$ Real-time imbalance bid of the community at time $t$, in scenario $\theta$
$\Delta \widetilde{c h}_{t, \theta, \pi}^{R T}, \Delta \widetilde{d c h}_{t, \theta, \pi}^{R T}$ Storage charging and discharging realtime deviations at time $t$, in scenario $\theta, \pi$

$\mathrm{a}_{t}^{D A} \quad$ Day-ahead market bid of the community at time $t$ $\mathrm{ch}_{t}^{D A}, d c h_{t}^{D A}$ Charging and discharging of the storage scheduled in the day-ahead stage for time $t$

$\mathrm{SoC}_{t}, \theta, \pi$ Storage state-of-charge at time $t$, in scenario $\theta, \pi$

\section{INTRODUCTION}

Energy communities (ECs) are envisioned to enhance the integration of distributed energy resources (DERs) into existing electricity markets, while incentivizing consumers and prosumers to unlock their flexibility potential [1]. In this paper, the joint coordination and market participation of several industrial sites, forming an EC, is modeled. The coordinating role is fulfilled by an energy community manager that performs a central optimization with partial knowledge of the coordinated asset's flexibility (leading to model uncertainty). The EC faces both load forecast and market price uncertainty. Moreover, for some loads, the characterization of the forecast uncertainty may be highly ambiguous. ${ }^{1}$. The different sites onsite flexibility can be represented as a battery storage system (BSS), complemented with a given inflexible residual load, reflecting the non-shiftable energy exchanges (Fig. 1), as done in, i.e., [2] for a set of thermostatically controlled loads. However, the state-of-charge (SoC) bounds of the approximating BSS model are subject to both parameter and model uncertainty. These uncertainties may be addressed by probabilistic constraints (most commonly by chance-constraints [3]) in stochastic optimization. CCs impose that the energy bounds are satisfied with a certain probability $\mathbb{P} \leq 1$, used, e.g., in [4], [5]. Assuming the several sources of multidimensional uncertainty $(\theta=\{1, \ldots, \Theta\}, \pi=\{1, \ldots, \Pi\})$, as used in the following of this research, the $\mathrm{CC}$ formulation reads as $\mathbb{P}(g(x, \theta, \pi) \leq 0) \leq(1-\epsilon) \forall t$. Note that the probability is measured on both $\theta$ and $\pi$ sets, but defined individually per time step $t$. CCs suffer from two drawbacks in the presented setting:

(1) This paper captures the underlying temporally correlated stochastic inputs via time-series scenarios. Chance constraints imposed on such correlated uncertainties typically only permit computationally expensive mixed-integer linear programming reformulations [6]. Authors of [7], [8] provide a tractable

\footnotetext{
${ }^{1}$ Distributional ambiguity is a concept for characterizing the limited knowledge about the exact underlying uncertainty, and is likely to faced by the EC when integrating new sites to the portfolio with limited historical measurements.
} 
numerical approximation, but this approach may lead to undesired levels of conservativeness for multidimensional uncertainties, as considered in this paper.

(2) Conceptually chance-constraints do not control the severity of the violation, allowing for infinitely large in-sample violations (discussed in Section III-B). The true violation cost of the considered distributed assets, e.g., thermal comfort constraints or undesired state-of-charge levels of electric vehicles, is usually unknown and time-varying. Hence, it may be highly beneficial to model energy bounds in a probabilistic framework that also imposes limitations on the violation magnitude.

Therefore, this paper builds on an alternative convex (conservative) approximation of $\mathrm{CCs}$, which is called the conditionalvalue-at-risk based constraint (CVaR-BC) [9], employed, e.g., in [10]-[13]. This formulation allows for considering both the probability and the severity, which is in finance often used for constraining the maximum risk of a portfolio selection problem [14], [15]. Furthermore, CVaR is a coherent risk measure [16], and thus can be recast as a convex linear programming problem when the corresponding function is linearly dependent on the decision variables [11], even if correlated time-series scenarios are used as inputs.

Generating correlated (temporally or spatially) scenarios from the available data is a non-trivial task requiring appropriate understanding of the underlying uncertain process(es), which in addition, may differ per distributed asset in an energy community. Therefore, the scenario sampling used in this paper, is based on an advanced shape-based clustering technique [17] applied on the existing scenario samples. This technique provides an adequate distance measure to compare time-series samples, for which, the traditional Euclidean-distance may fail to capture important characteristics.

Both CC and the presented CVaR-BC suffer from the limitation that if the forecast error distribution of a newly accommodated site in the community differs significantly from the ones of the managed portfolio, i.e., the community manager faces distributional ambiguity, the resulting model is likely to exhibit poor out-of-sample performance. To overcome the issue of over-fitting the model to the limited information of the portfolio [18], distributionally robust optimization (DRO) provides performance guarantees [19], [20]. However, implementation of distributionally robust chance-constraints (DROCCs) [19], [21] in approximation algorithms like [7], [8] is non-trivial and may be computationally challenging. To address the possible distributional ambiguity faced by the EC, a two-step modeling strategy is proposed. (i) In the first step, building on the shape-based distance measure, the error scenarios are clustered into multiple groups, forming an exogenous ambiguity set. (ii) Next, based on this information, a worst-case CVaR [22], [23] based constraint (WCVaR$\mathrm{BC}$ ) is implemented to enforce the CVaR-BC in all scenario groups which may describe the true uncertainty. This modeling property offers higher out-of-sample reliability, when distributional ambiguity is present in the EC's portfolio. A significant numerical advantage is that WCVaR-BC remains a convex and linear function (Section III), making it applicable for large scale power systems problems with temporally or spatially correlated uncertainty sets. In the presented research, a large number of forecast error scenarios are already available via onsite measurements, serving as potential scenario candidates.

The main contributions of this paper are summarized as:

1) The proposed tractable probabilistic constraint extends the works of [10]-[13] by using the worst-case CVaR function to incorporate distributional ambiguity into correlated time-series scenario driven stochastic optimization. The presented method is employed to enforce worstcase guarantees of the energy bound constraints (considering severity and frequency) within an energy community participating in both day-ahead and real-time energy markets. Complementary to the proposed models, an advanced shape-based clustering technique is implemented that better encapsulates temporal characteristics into the (dis)similarity measure among time-series scenarios.

2) First in a stylized example, the fundamental differences between $\mathrm{CC}$ and $\mathrm{CVaR}-\mathrm{BC}$, and then WCVaR-BC, are highlighted to motivate (i) the control of violation magnitudes, and (ii) the consideration of worst-case scenario sets. In the presented numerical studies the trade-off between violations and expected benefits are extensively analyzed, illustrating that WCVaR-BC shields significantly better against extreme outcomes, while leading to only modest average cost increase.

Note that all developed models are published at https:// github.com/Dmihaly/risky_community, to ease reproducibility and further developments.

\section{MODEL FORMULATION}

In this Section, the centralized scheduling model for the energy community is introduced. It is assumed that the community has access to both day-ahead (DA) energy and realtime (RT) imbalance markets, as shown in Fig. 1. The exchange with the imbalance market is considered to be limited to avoid the virtual trading of large amounts of electricity. The scheduling is performed by a price-taking energy community manager [24]-[26], which is responsible to pool the loads and their flexibility potential, thus mitigating the overall risk of the portfolio. First, a scalable formulation is presented to

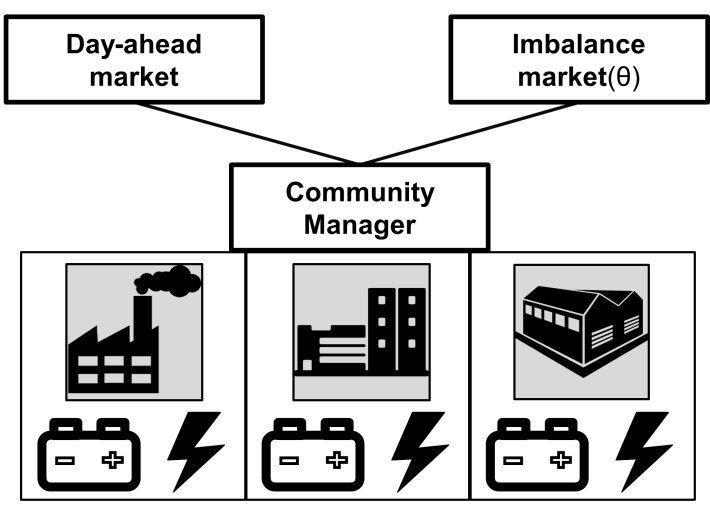

Fig. 1: Scheme showing the generic setting considered, involving of a community manager (CM) and multiple industrial sites with DERs. For the interaction with the markets two stages (DA, RT) are considered.

hedge the risk of infeasible schedules, based on CVaR-based 
probabilistic constraint. The approach is then extended to the worst-case CVaR based constraint to handle large ambiguity (e.g., for new clients with limited history). The resulting decision model is formulated as a stochastic scenario-based optimization program.

In our models, the DA market is considered as deterministic, while the imbalance market's outcome is stochastic, represented via a reduced number of time-series scenarios of imbalance price profiles. The price scenarios are obtained by clustering historical outcomes of the Belgian imbalance market. Likewise, the forecast errors associated with the industrial sites' load profiles are characterized by time-series scenarios. In particular, the clients are represented using real measurements from several industrial and commercial sites (collected by Schneider Electric [27]), including energy consumption, local production and corresponding forecast errors. The main focus of this research is on how these forecast errors can be managed using risk-based constraints on the state-of-charge limits.

The community manager (CM) optimizes the collective dayahead (here-and-now) and real-time (recourse) decisions $\mathcal{X}_{c m}$ $=\left\{x_{c m}^{D A}, \widetilde{x}_{c m}^{R T}\right\}$ belonging to the community, and the individual decision variables inheriting from the abstract flexibility model of each industrial site, i.e., DER agent $j \in \mathcal{J}$ on $\mathcal{X}_{\text {der }_{j}}=$ $\left\{x_{\text {der }}^{D A}, \widetilde{x}_{\text {der }}^{R T}\right\}$. The collection of DER agents is represented as an aggregated asset (der). The cost components of the objective function are defined as:

$$
\begin{aligned}
& C_{m}^{D A}\left(x_{c m}^{D A}\right)=\sum_{t \in \mathcal{T}} \lambda_{t}^{D A} \cdot a_{t}^{D A} \\
& \widetilde{C}_{m}^{R T}\left(\widetilde{x}_{c m}^{R T}\right)=\sum_{t \in \mathcal{T}} \sum_{\theta \in \Theta} P_{\theta} \cdot \widetilde{\lambda}_{t, \theta}^{R T} \cdot \Delta \widetilde{a}_{t, \theta}^{R T} \\
& C_{d e r}^{D A}\left(x_{c m}^{D A}, x_{d e r}^{D A}\right)=\sum_{t \in \mathcal{T}} O P E X^{D A} \cdot\left(d c h_{t}^{D A}+c h_{t}^{D A}\right) \\
& \widetilde{C}_{d e r}^{R T}\left(\widetilde{x}_{c m}^{R T}, \widetilde{x}_{d e r}^{R T}\right)= \\
& \sum_{t \in \mathcal{T}} \sum_{\theta \in \Theta} \sum_{\pi \in \Pi} P_{\theta} \cdot P_{\pi} \cdot O P E X^{R T} \cdot\left(\Delta \widetilde{d c h}_{t, \theta, \pi}^{R T}+\Delta \widetilde{c h}_{t, \theta, \pi}^{R T}\right)
\end{aligned}
$$

Eq. (1) and Eq. (2) denotes the sourcing cost of electricity from the DA energy market and the expected sourcing cost from the RT imbalance markets, respectively. The latter's uncertainty is captured via the scenario set $\theta$. Eq. (3) describes the operational expenditure associated with the activation of the distributed BSS at the day ahead stage, while Eq. (4) defines the same expected cost at the second, real-time stage, whose uncertainty is captured by the set $\pi$. The central optimization problem of the energy community (EC) reads as:

$$
\begin{aligned}
& \mathrm{EC}: \min _{\mathcal{X}_{\text {agg }}, \mathcal{X}_{\text {der }}} \mathcal{F}_{E C}=C_{m}^{D A}+\widetilde{C}_{m}^{R T}+C_{\text {der }}^{D A}+\widetilde{C}_{d e r}^{R T} \\
& \text { s.t. } \\
& \underline{a}^{D A} \leq a_{t}^{D A} \leq \bar{a}^{D A} \quad \forall t \\
& \underline{a}^{R T} \leq \Delta \widetilde{a}_{t, \theta}^{R T} \leq \bar{a}^{R T} \quad \forall t, \forall \theta \\
& a_{t}^{D A}+d c h_{t}^{D A}-c h_{t}^{D A}-d_{t}^{D A}-\Delta \tilde{d}_{t, \pi}^{R T} \\
& +\Delta \widetilde{a}_{t, \theta}^{R T}+\Delta \widetilde{d c h}_{t, \theta, \pi}^{R T}-\Delta \widetilde{c h}_{t, \theta, \pi}^{R T}=0 \quad \forall t, \forall \theta, \forall \pi \\
& 0 \leq d c h_{t}^{D A} \leq \bar{P} \quad \forall t \\
& 0 \leq c h_{t}^{D A} \leq \bar{P} \quad \forall t \\
& 0 \leq \Delta \widetilde{d c h}_{t, \theta, \pi}^{R T} \quad \forall t, \forall \theta, \forall \pi \\
& 0 \leq \Delta \widetilde{c h}_{t, \theta, \pi}^{R T} \quad \forall t, \forall \theta, \forall \pi \\
& d c h_{t}^{D A}+\Delta d c h_{t, \theta, \pi}^{R T} \leq \bar{P} \quad \forall t, \forall \theta, \forall \pi \\
& c h_{t}^{D A}+\Delta c h_{t, \theta, \pi}^{R T} \leq \bar{P} \quad \forall t, \forall \theta, \forall \pi \\
& \text { So } C_{t, \theta, \pi}=S o C_{t-1, \theta, \pi}+\eta^{c h} \cdot\left(c h_{t}^{D A}+\Delta \widetilde{c h}_{t, \theta, \pi}^{R T}\right) \\
& \text { - }\left(d c h_{t}^{D A}+\Delta \widetilde{d c h}_{t, \theta, \pi}^{R T}\right) / \eta^{d c h} \quad \forall t \backslash T, \forall \theta, \forall \pi \\
& \text { So } C_{t, \theta, \pi}=S o C_{1, \theta, \pi}+\eta^{c h} \cdot\left(c h_{t}^{D A}+\Delta \widetilde{c h}_{t, \theta, \pi}^{R T}\right) \\
& \text { - }\left(d c h_{t}^{D A}+\Delta \widetilde{d c h}_{t, \theta, \pi}^{R T}\right) / \eta^{d c h} \quad t=T, \forall \theta, \forall \pi \\
& 0 \leq \operatorname{SoC}_{t, \theta, \pi} \leq \bar{E} \quad \forall t, \forall \theta, \forall \pi
\end{aligned}
$$

Eq. (5b)-(5c) enforces that bids of the aggregator are within the predefined limits. This consideration reflects that in practice, the energy community is unlikely to perform virtual bidding with large amounts of electricity, i.e., its bids are limited by the capacity of the available assets. Eq. (5d) is the energy balance constraint of the community. Constraints $(5 \mathrm{e})-(5 \mathrm{j})$ guarantee that both day-ahead and real-time charging and discharging decisions comply with the power bounds. Eq. (5k)-(5l) track the temporal evolution of the stored energy. Lastly, constraint (5m) ensures that the stored energy is within the technical bounds. These bounds, however, may be highly uncertain and we therefore relax their strict enforcement in Section III.

\section{Probabilistic CONSTRAints}

First, the data pre-processing strategy and its connection to the uncertainty characterization is introduced (in Section III-A). Then, in Section III-B and III-C the CVaR and WCVaR based constraints are introduced and their properties are discussed qualitatively.

\section{A. Clustering and its connection to the model formulation}

The time-series scenarios, capturing the underlying uncertainty of the load were constructed by assembling empirical daily forecast errors from an extensive data set published by Schneider Electric (SE), containing historical electric load profiles of 70 industrial sites with the corresponding rollinghorizon forecasts, inspired by [28].

As several sites are included in the data set with different magnitudes of loads, the relative (to the day-ahead forecast) 
real-time load deviation, i.e., the relative forecast error, is calculated as:

$$
\text { relative deviation }=\frac{d_{t}^{D A}-\Delta \tilde{d}_{t, \pi}^{R T}}{d_{t}^{D A}}
$$

The collection of these forecast errors serves as the input for the scenario-based stochastic optimization, and initialized by the following strategy:

1) The forecast error scenarios are ordered into $K$ clusters based on their similarity/dissimilarity for each site $i \in I$.

2) Assuming each cluster $K=\left\{K_{1}, \ldots, K_{N}\right\}$ captures a particular trend of the uncertain process, i.e., different underlying distributions, $N$ sequences are selected from each cluster, resulting in $\Pi=N \cdot|K|$ scenarios.

In the clustering step, we use a shape-based distance measure proposed in [17], and implemented in [29], to characterize the similarity/dissimilarity between time-series forecast error scenarios. Mentioned in [30], the employed shape-based clustering accounts for the temporal correlation in the data and is less sensitive to scale, noise and time-shifts. The dissimilarity measure used in the clustering may be also utilized to ex-ante control the distance between the $K$ scenario clusters, leading to adjusted robustness in the optimization.

\section{B. Comparing CVaR-BC and $C C$}

This Section provides insights into the fundamental differences and similarities between chance constrains (CCs), i.e., value-at-risk (VaR) based constraints and the proposed CVARBC. For this purpose we connect the VaR and CVaR functions through a stylized example. A more in-depth analytical comparison of the two functions, and the resulting constraints may be found in [14], [31].

Figure $2 \mathrm{a}$ depicts a hypothetical probability distribution, where the confidence level is set to 0.8 for the probabilistic constraint, and 10 scenarios are considered. The height of each column corresponds to the probability of the scenarios. Furthermore, Fig. 2b shows two alternative shapes at the tail of the distribution via differentiated probabilities. Fig. 2 will be used in the following to describe the differences between VaR and CVaR.

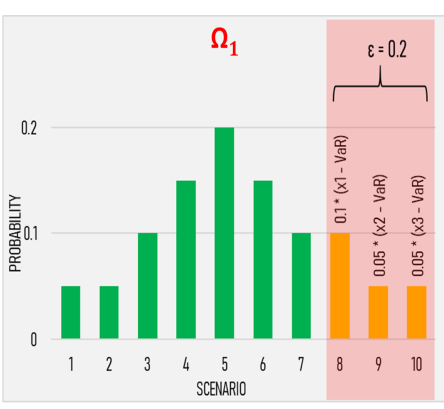

(a) Aggregated

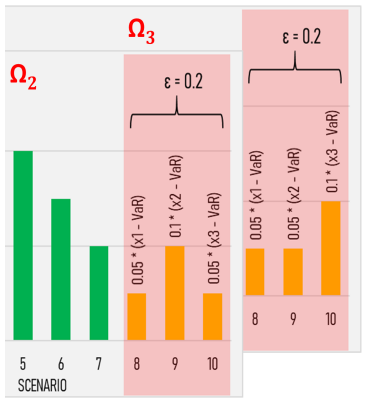

(b) Differentiated tails
Fig. 2: Green columns on the left-hand-side refer to the scenarios within the confidence interval characterized by $1-\epsilon$, i.e., no constraint violation allowed. In the orange scenarios violations are allowed. (a) VaR's and CVaR's positions are shown for comparison sor a single distribution $\Omega_{1}$ (b) indicates the changes for CVaR if different tails are considered in the alternative distributions $\Omega_{2}, \Omega_{3}$.
To introduce the VaR and CVaR, a convex function $g(x, \omega)$ is used, where $x \in X$ are the set of decision variables, and $\omega \in \Omega$ composed of $\pi \in \Pi, \theta \in \Theta$ are the set of stochastic scenarios. The VaR for the upper $(1-\epsilon)$-quantile of the joint bivariate distribution $(\Omega)$ is formulated as:

$$
\overline{\operatorname{VaR}}(\epsilon, x, \omega)=\inf _{\bar{\eta}}\{\bar{\eta}: \mathbb{P}(\omega \mid g(x, \omega)<\bar{\eta}) \leq(1-\epsilon)\}
$$

For lower (1- $\epsilon)$-quantile, VaR reads as:

$$
\underline{\operatorname{Va} R}(\epsilon, x, \omega)=\sup _{\underline{\eta}}\{\underline{\eta}: \mathbb{P}(\omega \mid g(x, \omega)<\underline{\eta}) \leq(1-\epsilon)\}
$$

where $\epsilon \in[0,1]$

$\overline{V a R}$ in Eq. (7a) is the largest value of $\eta$ guaranteeing that the probability of having a function value greater than $\eta$ is lower than or equal to $(1-\epsilon)$. Constraining the VaR function as well as using CCs guarantees that in the green scenarios of Fig. 2, defined by an exogenous confidence level $(1-\epsilon)$, no in-sample violation will occur. On the other hand, in the violating, orange scenarios, any level of violation is allowed by this formulation. Note that the three different distributions $\left(\Omega_{1}, \Omega_{2}, \Omega_{3}\right)$ are identical through the lens of the VaR function, i.e., CCs or VaR-based constraints are indifferent w.r.t. shape of the tail.

Contrary to Eq. (7a) and Eq. (7b), the CVaR characterizes the mean function value of the instances exceeding the VaR, i.e., the expected value of the scenarios at the tail (orange scenarios). Incorporating the degree of violation of scenarios and their corresponding probabilities, may lead to more restrictive outcomes compered to CCs. Therefore, CVaR-BC may be seen a convex approximation of the $\mathrm{CC}$ [32].

Following the formulation developed in [33], [34], the upper and lower CVaR functions are defined as:

$$
\overline{C V a R}(\epsilon, x, \omega)=\inf _{\bar{\eta}}\left\{\bar{\eta}+\frac{1}{1-\epsilon} \mathbb{E}[g(x, \omega)-\bar{\eta}]^{+}: \bar{\eta} \in \mathbb{R}\right\}
$$

$$
\underline{C V a R}(\epsilon, x, \omega)=\sup _{\underline{\eta}}\left\{\underline{\eta}-\frac{1}{1-\epsilon} \mathbb{E}[\underline{\eta}-g(x, \omega)]^{+}: \underline{\eta} \in \mathbb{R}\right\}
$$

where $[t]^{+}=\max (t, 0)$. Elimination of the plus function, as in [34], leads to the following linear programming forms:

$$
\begin{aligned}
& \overline{\operatorname{CVaR}}^{*}(\epsilon, x, \omega)= \\
& \bar{\eta}+\frac{1}{1-\epsilon} \mathbb{E}\{\delta(\omega): \delta(\omega) \geq(g(x, \omega)-\bar{\eta})\} \\
& \underline{\operatorname{CVaR}}{ }^{*}(\epsilon, x, \omega)= \\
& \underline{\eta}-\frac{1}{1-\epsilon} \mathbb{E}\{\delta(\omega): \delta(\omega) \geq(\underline{\eta}-g(x, \omega))\}
\end{aligned}
$$

where $\delta \in \mathbb{R}^{+}$is an auxiliary variable. The above simplification leads to significant computational advantages when modeling CVaR, compared to $\mathrm{VaR}$ or $\mathrm{CC}$, making it easily suitable for multi-source time-series scenario inputs. Using the above functions, we recast the energy content constraints Eq. 
(5m) of the BSS as upper or lower conditional-value-at-risk constraints.

$$
\begin{aligned}
& \operatorname{SoC}(x, \omega) \leq \bar{E} \Leftrightarrow \overline{C V a R}_{\text {SoC }}^{*}(\epsilon, x, \omega) \leq \bar{E} \\
& \operatorname{SoC}(x, \omega) \geq 0 \Leftrightarrow \underline{C V_{a} R_{S o C}^{*}(\epsilon, x, \omega) \geq 0}
\end{aligned}
$$

The fact that CVaR-BC can be modeled by a convex function, implies that it results in continuously increasing/decreasing, monotone in-sample expected objective values and violations. In contrast, $\mathrm{VaR}$ (and $\mathrm{CC}$ ) typically exhibit discrete jumps w.r.t. the confidence level. Such jumps may translate into inconsistent out-of-sample performance and expected outcomes can be significantly sensitive to the chosen confidence level. The above characteristics of the CVaR function make it particularly beneficial over $\mathrm{CC}$ in problems where violation costs are hard to be characterized ex-ante, e.g., for thermal discomfort (discussed in [35]).

In Table I the different expected violations are calculated, resulting from the alternative tails of Fig. 2a and Fig. 2b. The assumed violations for Scenarios $\{8,9,10\}$ are $\{1.0,1.5,2.0\}$. It can be noted that in the three different cases the modeler accepts very different levels of expected violations, despite the fixed violation level, which is ignored in the formulation of CCs. On the other hand, in the CVaR-BC this property is well-captured and can be compensated, e.g., by choosing lower violation levels in the more probable scenarios.

TABLE I: Expected and average violation in the violating scenarios (at the tail of the distributions), i.e., CVaR, for the three different distributions. The expected violation is calculated by the product of the absolute violation and the corresponding scenario's probability. The violations for Scenarios $\{8,9,10\}$ are $\{1.0,1.5,2.0\}$.

\begin{tabular}{|l|lll|}
\hline & \multicolumn{3}{|c|}{ Distributions } \\
\hline & $\Omega_{1}$ & $\Omega_{2}$ & $\Omega_{3}$ \\
Scenario 8 & $0.1 * 1.0$ & $0.05 * 1.0$ & $0.05^{*} 1.0$ \\
Scenario 9 & $0.05 * 1.5$ & $0.1 * 1.5$ & $0.05 * 1.5$ \\
Scenario 10 & $0.05 * 2.0$ & $0.05 * 2.0$ & $0.1 * 2.0$ \\
CVaR per tail & 0.275 & 0.3 & 0.325 \\
Avg. CVaR & \multicolumn{4}{|c}{0.3} \\
\hline
\end{tabular}

\section{Comparing WCVaR-BC and CVaR-BC}

Opposed to the CVaR case, in which $\Omega_{1}, \Omega_{2}, \Omega_{3}$ belong to the same scenario cluster $(k=1)$, the worst-case CVaR (WCVaR) function is used to allow for differentiation among a collection of scenario clusters $\left(\Omega^{k}, k \in \mathcal{K}\right)$, e.g. $k=1,2,3$ clusters can be characterized for $\Omega_{1}, \Omega_{2}, \Omega_{3}$. As a result, instead of aggregating the three tail approximations, the probabilistic constraint enforcement can be done for the worst one ( $\Omega_{3}$ in the stylized example, as shown in Table I). This way one can avoid over-fitting the model to the aggregation of all tails and reduce possible exposure to extreme outcomes. For a collection of scenario sets $\left(\Omega^{k}, k \in K\right)$, the WCVaR function is defined as the CVaR belonging to the worst realization:

$$
\begin{aligned}
& \overline{W C V a R}(\epsilon, x, \omega) \triangleq \sup _{k \in K} \overline{C V a R}^{k}\left(\epsilon, x, \omega^{k}\right) \\
& \underline{W C V a R}(\epsilon, x, \omega) \triangleq \inf _{k \in K} \underline{C V a R^{k}}\left(\epsilon, x, \omega^{k}\right)
\end{aligned}
$$

It is shown in [22] that WCVaR remains a coherent risk measure. Furthermore, the same linear approximation may be used for WCVaR, as derived for CVaR in [9], under the assumption that $f(x, \omega)$ is linear w.r.t. $x$, and $X$ is a convex polyhedron. Consequently the resulting $\mathrm{SoC}$ constraints may be written as a worst-case constraint that holds for all CVaR-s, belonging to set $K$ :

$$
\begin{array}{ll}
\overline{C V a R}_{\text {SoC }}^{k} \leq \overline{W C V a R}_{S O C} \leq \bar{E} & \forall k \in K \\
\underline{C V a R}_{\text {SoC }}^{k} \geq \underline{W C V a R}_{S O C} \geq 0 & \forall k \in K
\end{array}
$$

In the model formulation, $\mathrm{CVaR}$ is defined over the set of scenario clusters $K$, as defined in Section III-A, allowing for differentiation in its values. Then the worst-case CVaR (WCVaR) function [22], [23] is used to endogenously account for the worst realization of CVaR. Note, that when the model is supplied by a single cluster of scenarios $(k=1), \mathrm{CVaR}$ and WCVaR are identical.

\section{Numerical Results}

In this section, we evaluate the performance of our proposed WCVaR constraints w.r.t. CVaR-BC in modeling the energy bounds of the flexibility providers. In particular, the model is tested on an energy community composed of 5 industrial loads (denoted as MIX), referred to as $\{19,35,40,58,62\}$, randomly selected from the Schneider Electric dataset [27]. First, the clustering technique, described in Section III-A, is applied to construct the representative load error scenarios. We make the assumption that each of the 5 sites belongs to a different underlying distribution $\Omega^{k}$, where $k=1,2,3,4,5$. For each site, the distribution is modelled through sequences that are collected from all weekdays in the time-horizon of three months, from January to March in 2016, which results in 65 sequences for each site. These sequences are clustered into 13 clusters for each site. In each cluster, the closest element (defined by using the shape-based distance function [17]) to the centroid is selected as a prototype. The assigned probability of occurrence is proportional to the size of the cluster from which the prototype is selected. Lastly, each cluster's prototype and its probability of occurrence is moved to the final set of in-sample scenarios, leading to overall 65 scenarios (13 representative sequences for the 5 industrial clients). When using CVaR-BC no differentiation is made based on which site was the root of a given scenario, whereas this information is preserved in WCVaR to make distinction among the $k \in K$ scenario clusters, i.e., $k \in K$ underlying distributions.

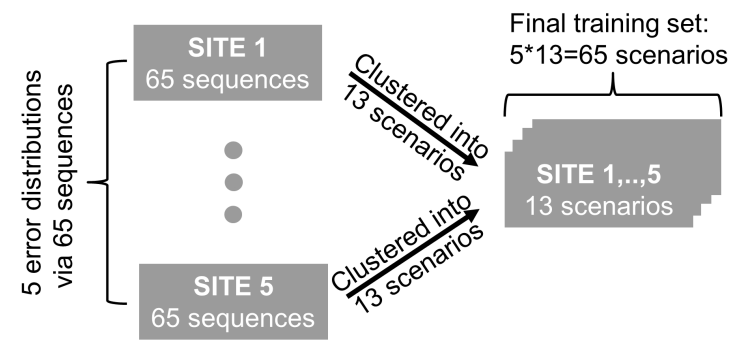

Fig. 3: Clustering strategy to define the in-sample scenarios. On the left, the collected error sequences for each 5 site is indicated. Via reducing each 5 site's 65 sequences to 13 clusters, we attain $5 \cdot 13$ overall in-sample scenarios. 
We first introduce the data used in the numerical case studies (Section IV-A). Then, in Section IV-B, we compare the out-of-sample reliability (obtained by CVaR-BC and WCVaR$\mathrm{BC}$ ). Lastly, we highlight the mean as well as the maximum violations in function of the corresponding objective value (Section IV-C).

\section{A. Experimental data}

In the case studies, we assume that the only varying (uncertain) parameters are the real-time load realizations (forecast error with respect to day-ahead expectations), whereas the dayahead forecasted load is used from a single day to focus the analysis on the effects of uncertainty. Furthermore, for the sake of simplicity, we model a single site assuming that it represents the aggregation of several DERs both in terms of fixed load and flexibility. ${ }^{2}$ The modeled BSS, which acts as a surrogate for the flexible part of the load, has $3 \mathrm{MW}$ of charging and discharging power (with $98 \%$ round-trip efficiency) and 0.4 MWh energy capacity. The daily price profiles were downloaded from ELIA's [36] (the Belgian Transmission System Operator) website. The DA market price is deterministic, and its average value over the day is $\bar{\lambda}_{t}^{D A}=18.2 € / \mathrm{MWh}$. and the expected average RT imbalance market price is $\mathbb{E}\left(\overline{\widetilde{\lambda}}_{t, \theta}^{R T}\right)=$ $18.6 € / \mathrm{MWh}$. To model the uncertainty of real-time electricity prices, 5 scenarios are considered in the optimization, obtained by clustering the yearly data into the 5 representative clusters and selecting their prototypes. Similarly to the DA forecasted part of the demand, the deterministic DA market price and the stochastic RT market price scenarios $(\theta \in \Theta)$ are not altered in the simulations. To avoid unrealistic exchanged quantities with the markets, and extensive virtual bidding, the DA market position of the community is limited to two times the maximal forecasted load (Eq. (5b)), whereas the RT market position is bounded by half of the maximal load deviation (Eq. (5c)) in all considered scenarios. The operational expenditure (OPEX) of the DER's flexibility is $0.01 \cdot \bar{\lambda}_{t}^{D A}$ in the DA stage and $0.05 \cdot \mathbb{E}\left(\widetilde{\lambda}_{t, \theta}^{R T}\right)$ in the RT recourse stage in $€ / \mathrm{MWh}$. The higher RT OPEX intends to reflect the increasing communication and scheduling burden when executing deviations closer to realtime.

In the in-sample optimization, the stochastic model is supplied with all the input data such as the DA and RT market prices (with 5 scenarios), the deterministic (fixed) DA forecasted load profile and the generated RT forecast error scenarios (65 scenarios). The 65 forecast error scenarios are constructed by applying the clustering technique (as presented in Section III-A) on the in-sample set of sites: $\{19,35,40$, $58,62\}$. Once optimality is reached, all DA decisions and the RT imbalance market positions are fixed in the optimization model used in the test runs. In the test runs the RT forecast error realization is being updated, which is the only altered input. Out-of-sample feasibility is not guaranteed due to the (i) in-sample violations of the probabilistic SoC constraints (Eq. 5m), (ii) the DA decisions and the RT imbalance market

\footnotetext{
${ }^{2}$ This is solely a modeling simplification and the framework would allow for the inclusion of multiple sites.
}

positions are fixed to the training model's outcome, i.e., only RT charging and discharging decisions are re-optimized, while the load forecast error takes different values. To ensure that the model is out-of-sample feasible, an ancillary slack variable $\left(s^{U P}, s^{D O W N}\right)$ is added to the SoC bounds (Eq. $\left.5 \mathrm{~m}\right)$. The non-zero values of the slack variables are penalized in the objective function with a large violation coefficient (1000), leading to the following extension of the objective function: $\sum_{t, d}$ penalty $\cdot\left(s_{t, d}^{U P}+s_{t, d}^{D O W N}\right)$. The penalty cost is not accounted for in the reported objective values.

Test simulations are conducted on 7 new sites $\{12,58$, $40,17,70,68,29\}$, which were not part of the training set. For each site, 65 days are simulated per in-sample confidence level, leading to 650 days considering all 10 confidence levels. The updated parameter is the daily forecast error, i.e., the uncertain part of the demand which was also collected from the Schneider Electric dataset from January 2016 to March 2016.

\section{B. In- and out-of-sample reliability comparison of CVaR-BC and WCVaR-BC}

Fig. 4 depicts the out-of-sample reliability in function of the in-sample confidence level. The reliability is calculated based on the total number of violating instances, i.e., cases in which $s_{t, d}^{U P}, s_{t, d}^{D O W N}$ are non-zero were enumerated in all time steps $(t \in T)$ and in all simulated days $(d \in D)$, and the total instances, i.e., number of time steps multiplied by the number of days (31200). The results indicate that WCVaR-BC (indicated by circle markers) leads to always higher reliability than CVaR-BC (triangle markers) for all subject sites. Although, the difference is more pronounced for some sites (e.g. for 70, 12), WCVaR's higher reliability is an expected outcome given the more conservative nature of the WCVaR function.

A factor of crucial importance influencing the out-of-sample performance is the difference between the inputs used in the out-of-sample simulations and the inputs used in the training (in-sample) phase of the optimization. Obviously one can expect better performance if the in-sample uncertainty approximation is closer to the realized inputs. When selecting the test sites, it was taken into consideration that the test set should involve various samples w.r.t. their closeness to the insample data. To characterize the (dis)similarity between insample and out-of-sample instances, the same shape-based distance was used as in the clustering step for scenario reduction. In Fig. 5 it is shown how the average ${ }^{3}$ sequence of a few selected test site compares to the average sequence of the in-sample training MIX. It is visible that site 12 is the most similar whereas 29 has the highest dissimilarity. The quantified distances between the prototype of each test site and the training set are summarized in Table I, confirming that site 12 is indeed the closest match, whereas site 29 is one of the highest dissimilarity. The possible correlation of these distances to the changes in the out-of-sample performance, e.g,

\footnotetext{
${ }^{3}$ The average instance is calculated by using the shape extraction function of the shape-based clustering method.
} 


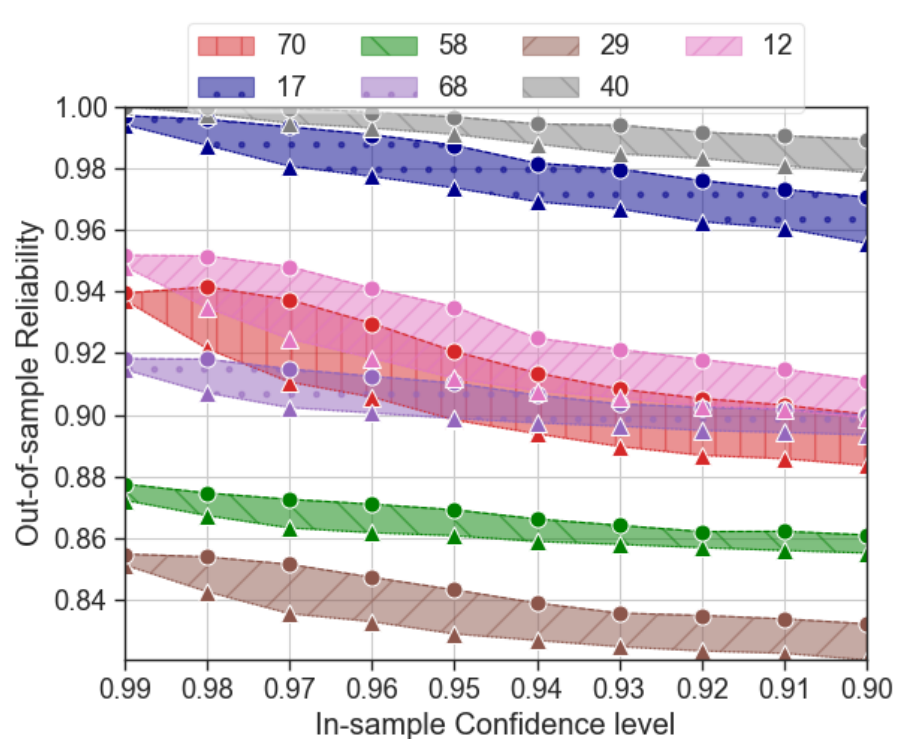

Fig. 4: The reliability, calculated based on the number of violating instances in the test runs attained under the given in-sample confidence levels. Triangle markers indicate the results of the CVaR-BC and circles the outcomes resulted from the WCVaR-BC. The numbers in the legend refer to the test site's id.

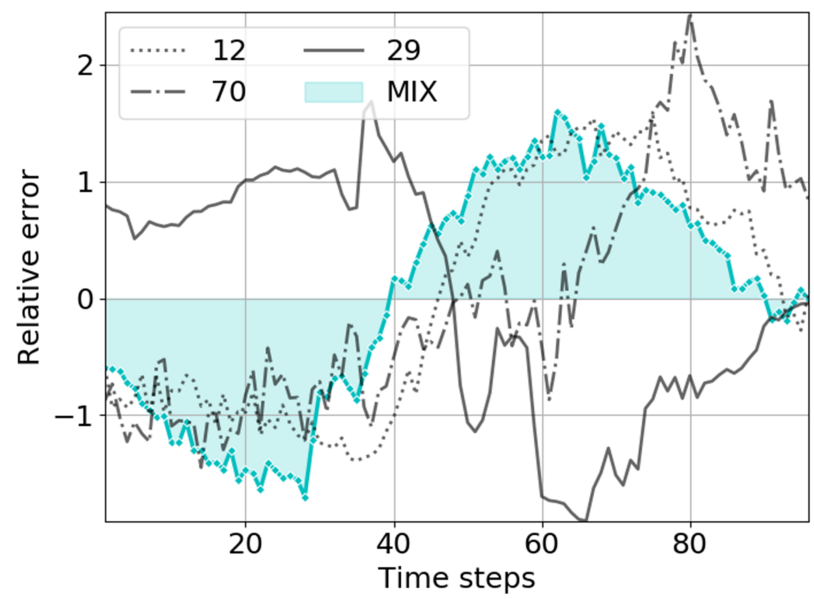

Fig. 5: The extracted prototypes using the shape extraction function of [29]. MIX indicates the prototype of the in-sample training set, whereas $12,29,70$ refer to the prototype of three test sites.

in the number of violations for the probabilistic constraints are discussed later in this Section.

TABLE II: The distance between each site's prototype and the mean sequence of the in-sample scenario MIX, calculated based on the shape-based distance.

\begin{tabular}{llllllll}
\hline Site & 12 & 58 & 40 & 17 & 70 & 68 & 29 \\
\hline Distance & 0.055 & 0.159 & 0.258 & 0.294 & 0.268 & 0.510 & 0.501 \\
\hline
\end{tabular}

\section{The trade-off between violations and expected benefits}

In Fig. 6, in the left y-axis the mean daily operation cost is plotted, calculated by the following function: $\frac{1}{N_{d}} \cdot \sum_{t, d} \mathcal{F}_{t, d}^{E C}$, where $N_{d}$ refers to the number of simulated days for each confidence level. Note that the aggregator makes profit in its daily operation. However, here the negative costs are plotted to better visualize their comparison with the mean daily violations: $\frac{1}{N_{d}} \cdot \sum_{t, d}\left(s_{t, d}^{U P}+s_{t, d}^{D O W N}\right)$, shown on the right y-axis.

The WCVaR-BC by definition imposes more conservative constraints such that the higher reliability comes at the cost of lower expected mean performance. This can be understood by looking at the projections in Fig. 6. If e.g., one compares the cost obtained by WCVaR-BC at $\epsilon=0.08$, for site 12 a similar cost can be obtained at $\epsilon=0.03$ by CVaR-BC. The mean violations (indicated by the red $\Delta$ sign), however, differ significantly at the chosen confidence levels, despite that costs are close to each other. The difference is showing that WCVaR-BC may lead to higher average violation when calibrated to achieve similar objective values as CVaR-BC. The same projections, made for site 29 , show a much smaller difference, which may be explained by the different distances from the training scenario MIX. It is also visible that in lower confidence levels, the the operational costs are converging, whereas the corresponding violations remain much lower with WCVaR-BC.

The results of Fig. 6 suggests that CVaR-BC on average leads to higher reliability for a given average out-of-sample cost. However, as discussed in the motivation of WCVaR$\mathrm{BC}$, its advantage lies in the ability to reduce the exposure to extreme out-of-sample outcomes, which has the most benefit when limited historical information is available to approximate the uncertain process. The extreme outcomes, i.e., instances with the highest out-of-sample violations due to the wrong approximation of the underlying uncertainty, are not well depicted in the aggregated results of Fig. 4 and Fig. 6 . Therefore, it is insightful to assess the spread of the maximum violations as a function of the corresponding profit in each violating case (Fig. 7). This metric is of great relevance when choosing one of the proposed risk-based constraints over CC.

Figure 7 shows the bi-variate kernel density estimate (KDE) plot indicating the expected spectrum of the maximum observed daily violations and the corresponding profits for sites $\{12,70,29\}$. In addition, on the marginal $\mathrm{x}$ and $\mathrm{y}$ axis the histograms are plotted individually for the distribution of both maximum violations and profits. WCVaR-BC leads to lower violations and lower profits by definition. However, it was observed in the analysis that reducing the confidence level by $5-7 \%$ often leads to similar profits by WCVaR$\mathrm{BC}$ as by CVaR-BC. Therefore, to generate results from the same frontier in the comparison of maximum violations, the WCVaR-BC model was solved to lower confidence levels ( $\epsilon=0.01-0.17$, meaning $83 \%$ was the lowest confidence). The maximum violations are collected for each 65 real-time forecast error realizations and for each in-sample confidence level (\#10, \#17). Overall this leads to 650 possible violations with CVaR-BC and to 1105 with WCVaR-BC. Due to the difference in the number of studied instances, we normalized both the histograms and the KDE plots, such that their area always adds up to one.

Fig. 7 a shows that the advantage of using WCVaR-BC is the most prominent for site 29 , which is the second furthest 


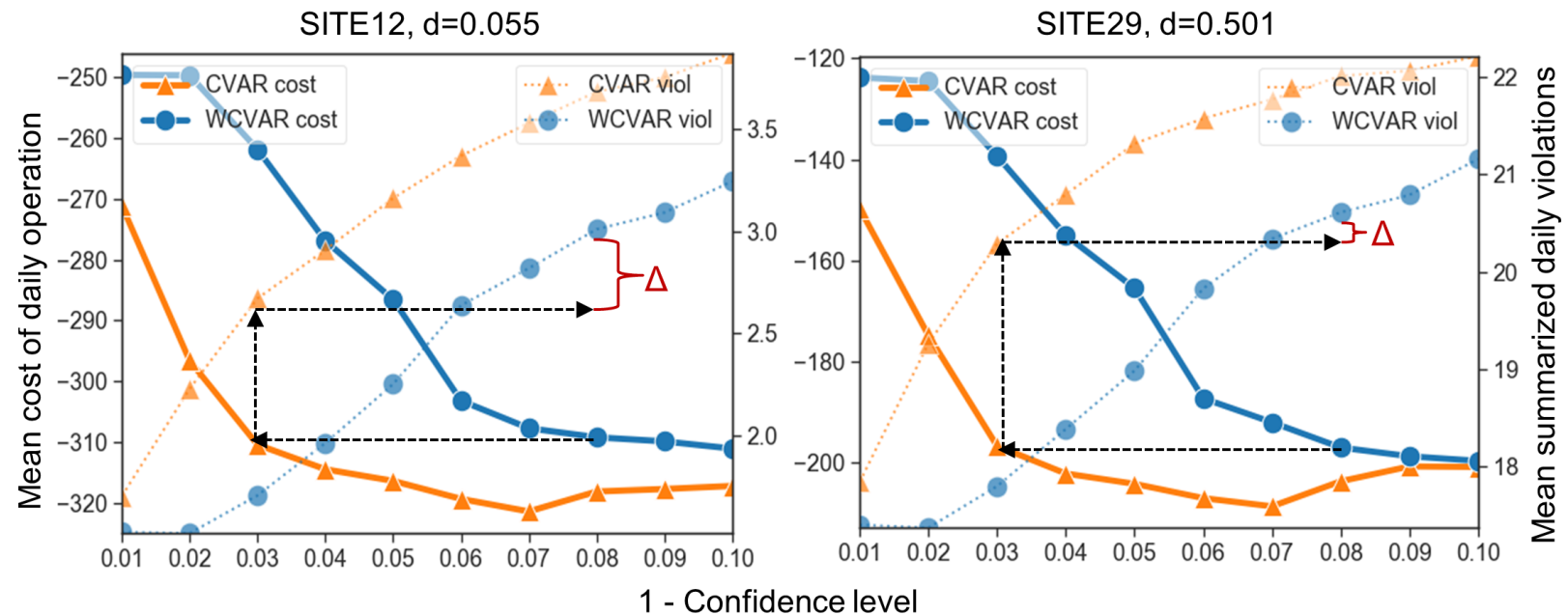

Fig. 6: Mean out-of-sample cost of daily operation (left-hand-side of the y-axis) and mean daily upper plus lower bound violations of the SoC constraint (right-hand-side of the y-axis) is plotted in function of the confidence level $(1-\epsilon)$. The black dashed rectangle indicates an example of comparing WCVaR-BC ${ }^{92 \%}$ to $\mathrm{CVaR}-\mathrm{BC}^{97 \%}$ in terms of daily operational cost and the mean daily violations. The red $\Delta$ sign highlights that the WCVaR-BC violation is higher corresponding to around the same operational cost as attained by CVaR-BC. The "d" letter on the top of each figure indicate the difference between the prototype of the in-sample mix and the test site.

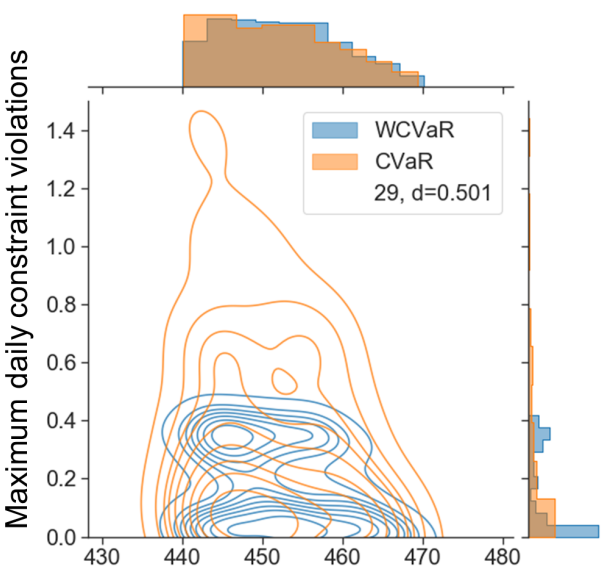

(a)

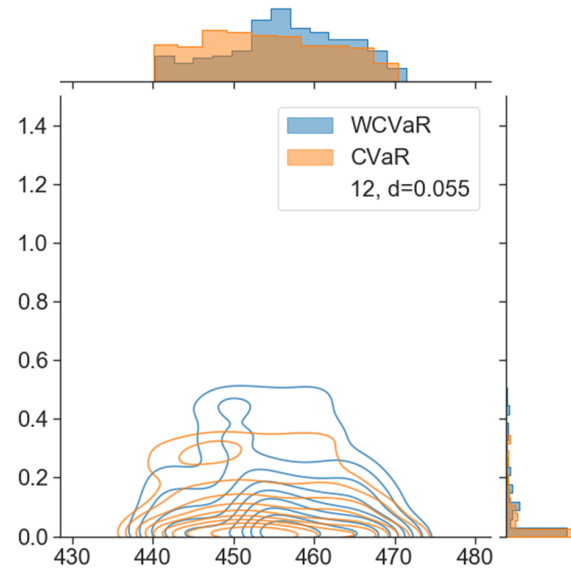

(b)

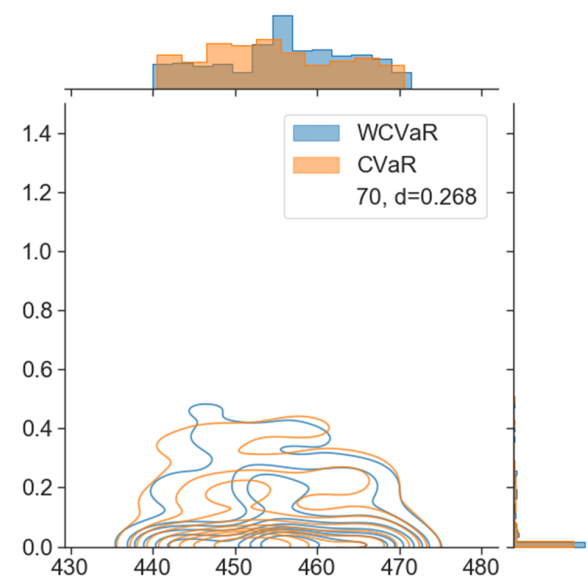

(c)

Daily profit

Fig. 7: In the center the kernel density estimation of the maximum daily violations and corresponding profits is shown, collected by solving CVaR-BC at $\epsilon=0.01-0.1$ and WCVaR-BC at $\epsilon=0.01-0.17$. In the marginal $\mathrm{x}$ and $\mathrm{y}$ axis the histograms for maximum violations and profits are plotted individually. From all the obtained results, we report the ones with the highest profit as they produce the highest violating instances. Therefore, the results were cut-off at the profit of $440 €$ for all sites. All plots are normalized such that their area individually adds up to one.

candidate from the in-sample scenario MIX (Table II). It can be observed that the maximum violations reach a more than two times higher level than for site 12 and 70 at the tail of the distribution (Fig. 7a marginal y-axis), while the profits are spread in around the same range (Fig. 7a marginal $\mathrm{x}$ - axis). For this site, the in-sample scenario set was a particularly inaccurate approximation, as such the severity of the maximum violating instances are large with $\mathrm{CVaR}-\mathrm{BC}$ that fits the model tighter to the training set. WCVaR-BC, on the contrary, was capable to circumvent such severe violations, remaining in the same range as for the other two sites (shown by the marginal $y$-axes of Fig. 7). Contrary to site 29, the outcomes of site 12 (Fig. 7b), the best match with the training set, indicate moderately lower maximum violations with $\mathrm{CVaR}-\mathrm{BC}$ compared to
WCVaR-BC. Site 70, which is representative for most other test sites not shown in the Fig. 7, does not show pronounced differences in using CVaR-BC or WCVaR-BC. The profits as well as the maximum violations are similarly distributed. The three different sites shown by Fig. 7 demonstrate well the trade-off faced by the modeler when choosing between the two proposed risk-based constraints.

\section{Conclusion And OUtLoOK}

This paper implements two data-driven risk-based constraints for the risk-aware probabilistic enforcement of the flexibility bounds of an energy community that aggregates a variety of distributed assets, and participates in day-ahead energy and imbalance markets. First, the CVaR-BC is formu- 
lated to account for both the severity and the probability of the violations when representing the energy bounds of the EC, which carry potential benefits over CCs. Next, the former constraint is extended to a novel WCVaR-BC that differentiates the $\mathrm{CVaR}$ value among the sub-clusters of clients, allowing to hedge against distributional ambiguity (inheriting from the varying nature of the on-site DER assets). The resulting timeseries scenario-driven optimization models can tackle largescale problem instances in a linear programming fashion.

After qualitatively comparing the proposed constraints to $\mathrm{CC}$, in a numerical analysis it was shown that WCVaR-BC allows for reducing the exposure to extreme violation levels via the, when assuming limited knowledge about the forecast errors. The proposed constraints can facilitate the flawless expansion of the EC's portfolio by assets with scarce historical data. Furthermore, the authors believe that the implemented modeling strategy may be well-suited for a broad range of power systems applications with ambiguous uncertainty sets and limited knowledge on the cost of constraint violations. Moreover, the convex nature of the risk-based constraints allow for interpreting the associated dual variables as prices in local energy markets. In future research, this property may ease the risk-aware trading of flexible resources.

\section{REFERENCES}

[1] T. Morstyn, N. Farrell, S. J. Darby, and M. D. McCulloch, "Using peer-to-peer energy-trading platforms to incentivize prosumers to form federated power plants," Nature Energy, vol. 3, no. 2, pp. 94-101, 2018.

[2] H. Hao, B. M. Sanandaji, K. Poolla, and T. L. Vincent, "Aggregate flexibility of thermostatically controlled loads," IEEE Transactions on Power Systems, vol. 30, no. 1, pp. 189-198, 2014.

[3] A. Charnes and W. W. Cooper, "Chance-constrained programming," Management science, vol. 6, no. 1, pp. 73-79, 1959.

[4] H. Zhang, Z. Hu, E. Munsing, S. J. Moura, and Y. Song, "Data-driven chance-constrained regulation capacity offering for distributed energy resources," IEEE Transactions on Smart Grid, vol. 10, no. 3, pp. $2713-$ $2725,2018$.

[5] J.-F. Toubeau, J. Bottieau, Z. De Greve, F. Vallee, and K. Bruninx, "Data-driven scheduling of energy storage in day-ahead energy and reserve markets with probabilistic guarantees on real-time delivery," IEEE Transactions on Power Systems, 2020.

[6] S. Ahmed and D. J. Papageorgiou, "Probabilistic set covering with correlations," Operations Research, vol. 61, no. 2, pp. 438-452, 2013.

[7] K. Margellos, P. Goulart, and J. Lygeros, "On the road between robust optimization and the scenario approach for chance constrained optimization problems," IEEE Transactions on Automatic Control, vol. 59, no. 8, pp. 2258-2263, 2014

[8] M. Vrakopoulou, B. Li, and J. L. Mathieu, "Chance constrained reserve scheduling using uncertain controllable loads part i: Formulation and scenario-based analysis," IEEE Transactions on Smart Grid, vol. 10, no. 2, pp. 1608-1617, 2017.

[9] R. T. Rockafellar, S. Uryasev et al., "Optimization of conditional valueat-risk," Journal of risk, vol. 2, pp. 21-42, 2000.

[10] Q. P. Zheng and P. M. Pardalos, "Stochastic and risk management models and solution algorithm for natural gas transmission network expansion and lng terminal location planning," Journal of optimization theory and applications, vol. 147, no. 2, pp. 337-357, 2010.

[11] Y. Huang, Q. P. Zheng, and J. Wang, "Two-stage stochastic unit commitment model including non-generation resources with conditional value-at-risk constraints," Electric Power Systems Research, vol. 116, pp. $427-438,2014$

[12] A. N. Madavan and S. Bose, "Risk-sensitive energy procurement with uncertain wind," in 7th IEEE Global Conference on Signal and Information Processing, GlobalSIP 2019. Institute of Electrical and Electronics Engineers Inc., 2019, p. 8969200.

[13] T. Summers, J. Warrington, M. Morari, and J. Lygeros, "Stochastic optimal power flow based on conditional value at risk and distributional robustness," International Journal of Electrical Power \& Energy Systems, vol. 72, pp. 116-125, 2015.
[14] G. J. Alexander and A. M. Baptista, "A comparison of var and cvar constraints on portfolio selection with the mean-variance model," Management science, vol. 50, no. 9, pp. 1261-1273, 2004.

[15] P. Krokhmal, J. Palmquist, and S. Uryasev, "Portfolio optimization with conditional value-at-risk objective and constraints," Journal of risk, vol. 4, pp. 43-68, 2002.

[16] F. Delbaen, "Coherent risk measures," Blätter der DGVFM, vol. 24, no. 4, pp. 733-739, 2000.

[17] J. Paparrizos and L. Gravano, "k-shape: Efficient and accurate clustering of time series," in Proceedings of the 2015 ACM SIGMOD International Conference on Management of Data, 2015, pp. 1855-1870.

[18] H. Yang, S. Zhang, D. Qiu, J. Zhao, M. Lai, Z. Y. Dong, and Z. H. Dong, "Distributionally robust optimal bidding of controllable load aggregators in the electricity market," IEEE Transactions on Power Systems, vol. 33, no. 1, pp. 1089-1091, 2017.

[19] S. Zymler, D. Kuhn, and B. Rustem, "Distributionally robust joint chance constraints with second-order moment information," Mathematical Programming, vol. 137, no. 1-2, pp. 167-198, 2013.

[20] P. M. Esfahani and D. Kuhn, "Data-driven distributionally robust optimization using the wasserstein metric: Performance guarantees and tractable reformulations," Mathematical Programming, vol. 171, no. 1-2, pp. 115-166, 2018.

[21] A. Arrigo, C. Ordoudis, J. Kazempour, Z. De Grève, J.-F. Toubeau, and F. Vallée, "Wasserstein distributionally robust chance-constrained optimization for energy and reserve dispatch: An exact and physicallybounded formulation," European Journal of Operational Research, 2021.

[22] S. Zhu and M. Fukushima, "Worst-case conditional value-at-risk with application to robust portfolio management," Operations research, vol. 57, no. 5, pp. 1155-1168, 2009.

[23] X. Tong, F. Wu, and L. Qi, "Worst-case cvar based portfolio optimization models with applications to scenario planning," Optimization Methods \& Software, vol. 24, no. 6, pp. 933-958, 2009.

[24] S. Han, S. Han, and K. Sezaki, "Development of an optimal vehicle-togrid aggregator for frequency regulation," IEEE Transactions on smart grid, vol. 1, no. 1, pp. 65-72, 2010.

[25] M. R. Sarker, Y. Dvorkin, and M. A. Ortega-Vazquez, "Optimal participation of an electric vehicle aggregator in day-ahead energy and reserve markets," IEEE Transactions on Power Systems, vol. 31, no. 5, pp. 35063515, 2015.

[26] K. Bruninx, H. Pandžić, H. Le Cadre, and E. Delarue, "On the interaction between aggregators, electricity markets and residential demand response providers," IEEE Transactions on Power Systems, vol. 35, no. 2, pp. 840-853, 2019.

[27] Schneider, Electric, "Microgrid energy management benchmark," https://data.exchange.se.com/explore/dataset/ microgrid-energy-management-benchmark-metadata/information/.

[28] T. Hong, P. Pinson, S. Fan, H. Zareipour, A. Troccoli, and R. J. Hyndman, "Probabilistic energy forecasting: Global energy forecasting competition 2014 and beyond," 2016.

[29] A. Sardá-Espinosa, "Time-series clustering in $\mathrm{r}$ using the dtwclust package," The R Journal, 2019.

[30] A. Sardá-Espinosa, "Comparing time-series clustering algorithms in $\mathrm{r}$ using the dtwclust package," $R$ package vignette, vol. 12, p. 41, 2017.

[31] S. Sarykalin, G. Serraino, and S. Uryasev, "Value-at-risk vs. conditional value-at-risk in risk management and optimization," in State-of-the-art decision-making tools in the information-intensive age. Informs, 2008, pp. 270-294.

[32] A. Nemirovski and A. Shapiro, "Convex approximations of chance constrained programs," SIAM Journal on Optimization, vol. 17, no. 4, pp. 969-996, 2007.

[33] R. T. Rockafellar, S. Uryasev et al., "Optimization of conditional valueat-risk," Journal of risk, vol. 2, pp. 21-42, 2000.

[34] R. T. Rockafellar and S. Uryasev, "Conditional value-at-risk for general loss distributions," Journal of banking \& finance, vol. 26, no. 7, pp. 1443-1471, 2002.

[35] N. Good, E. Karangelos, A. Navarro-Espinosa, and P. Mancarella, "Optimization under uncertainty of thermal storage-based flexible demand response with quantification of residential users' discomfort," IEEE Transactions on Smart Grid, vol. 6, no. 5, pp. 2333-2342, 2015.

[36] Elia NV, "Grid data," Available at http://www.elia.be/en/grid-data, 2020. 